\title{
Smart $\mathrm{Ti}_{3} \mathrm{C}_{2} \mathrm{~T}_{\mathrm{x}}$ MXene Fabric with Fast Humidity Response and Joule Heating for Healthcare and Medical Therapy Applications
}

\author{
Xing Zhao ${ }^{1}$, Li-Ya Wang ${ }^{2}$, Chun-Yan Tang ${ }^{1}$, Xiang-Jun Zhal, Yong Liu ${ }^{3}$, Bai-Hai Su ${ }^{2}$ Kai Ke ${ }^{*}$, \\ Rui-Ying Bao ${ }^{1}$, Ming-Bo Yang ${ }^{1}$, Wei Yang ${ }^{1 *}$
}

${ }^{I}$ College of Polymer Science and Engineering, Sichuan University, State Key Laboratory of Polymer Materials Engineering, Chengdu, 610065, Sichuan, China.

${ }^{2}$ Department of Nephrology, West China Hospital, Sichuan University, Chengdu, 610041, China.

${ }^{3}$ Science and Technology Information Center, PetroChina West East Gas Pipeline Company, Wuhan, 430074, Hubei, China.

Xing Zhao, Li-Ya Wang and Chun-Yan Tang contribute equally to this work.

*Corresponding authors: kaike@scu.edu.cn (K. Ke) and weiyang@scu.edu.cn (W. Yang)
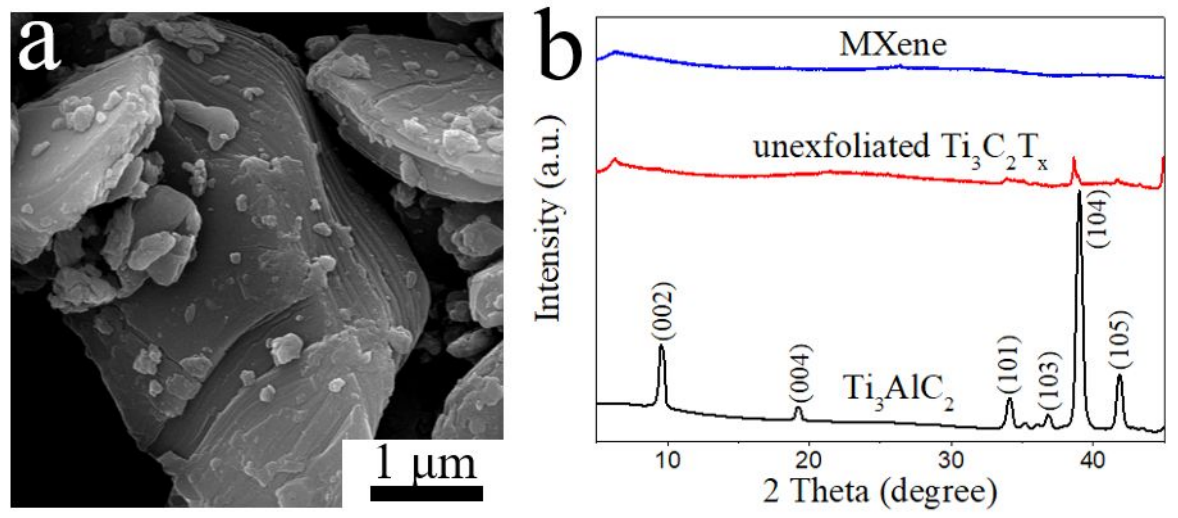

Figure S1. (a) SEM image of $\mathrm{Ti}_{3} \mathrm{AlC}_{2}$. (b) XRD patterns of $\mathrm{Ti}_{3} \mathrm{AlC}_{2}$, unexfoliated $\mathrm{Ti}_{3} \mathrm{C}_{2} \mathrm{~T}_{\mathrm{x}}$, and delaminated $\mathrm{Ti}_{3} \mathrm{C}_{2} \mathrm{~T}_{\mathrm{x}}$ (MXene). 


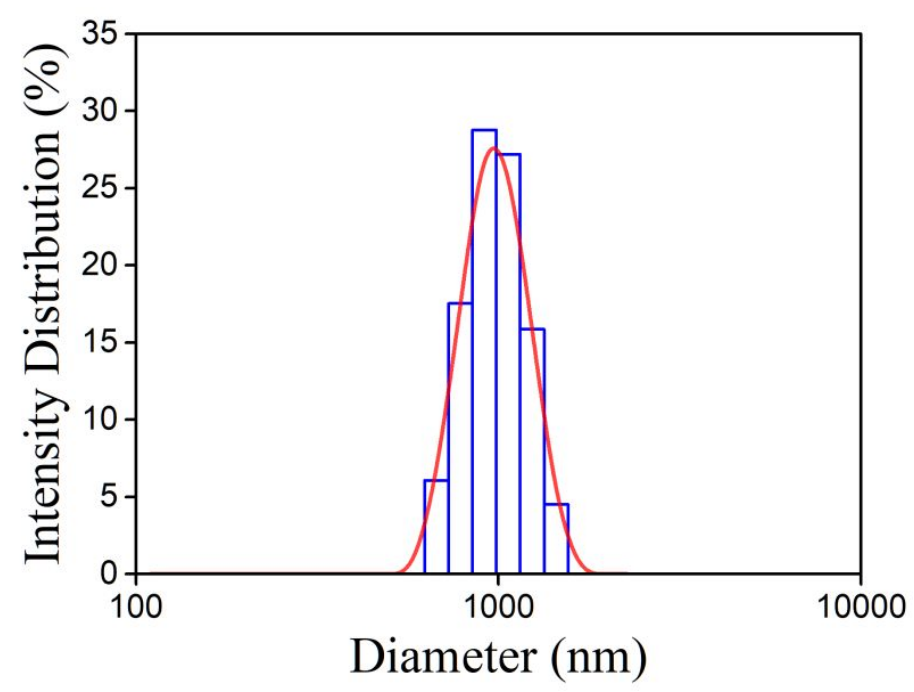

Figure S2. Flake size distribution of MXene dispersion.
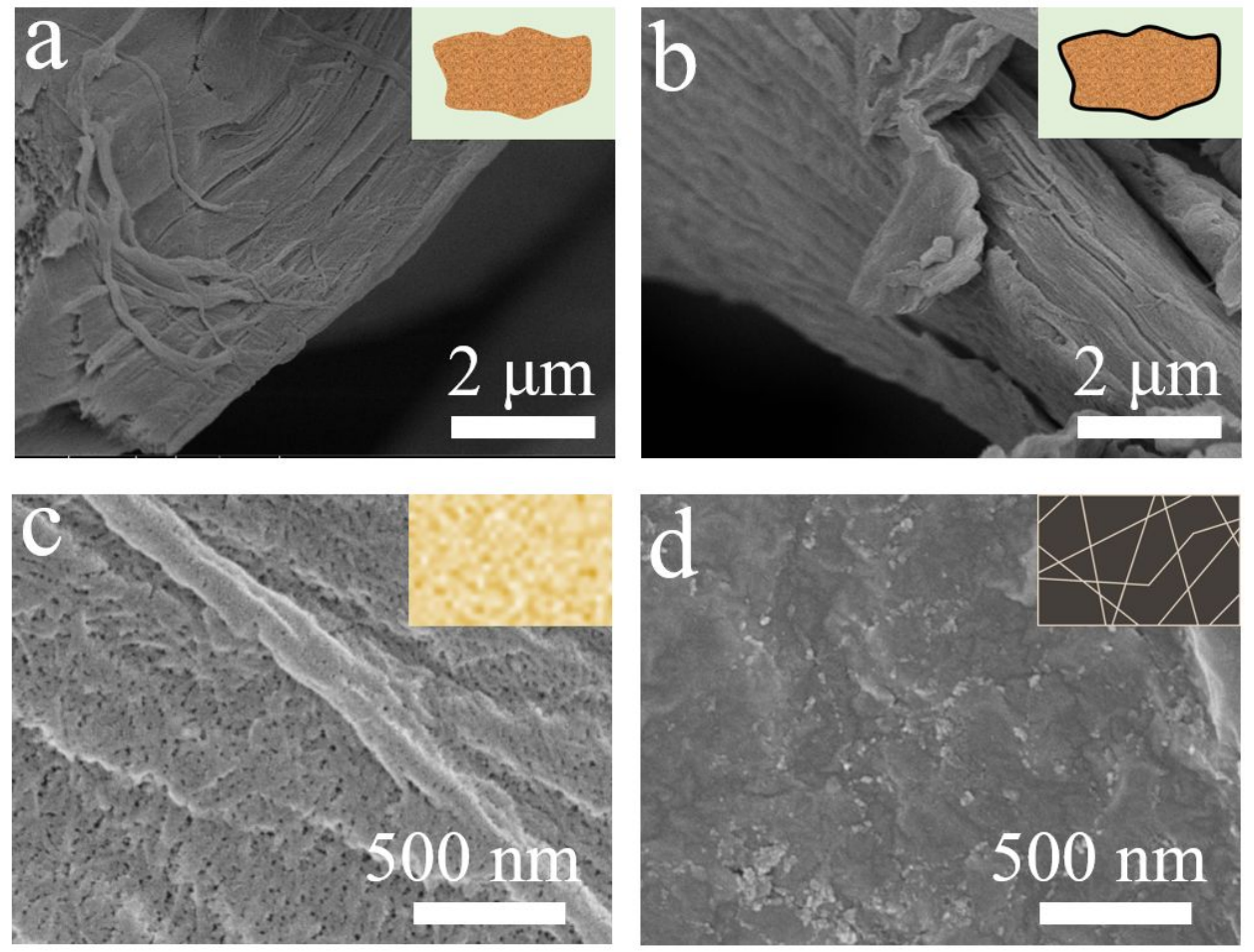

Figure S3. Side-view SEM images under higher magnifications of (a) the cellulose fabric and (b) the M-fabric. Surface-view SEM images under higher magnifications of (c) cellulose fabric and (d) M-fabric. 


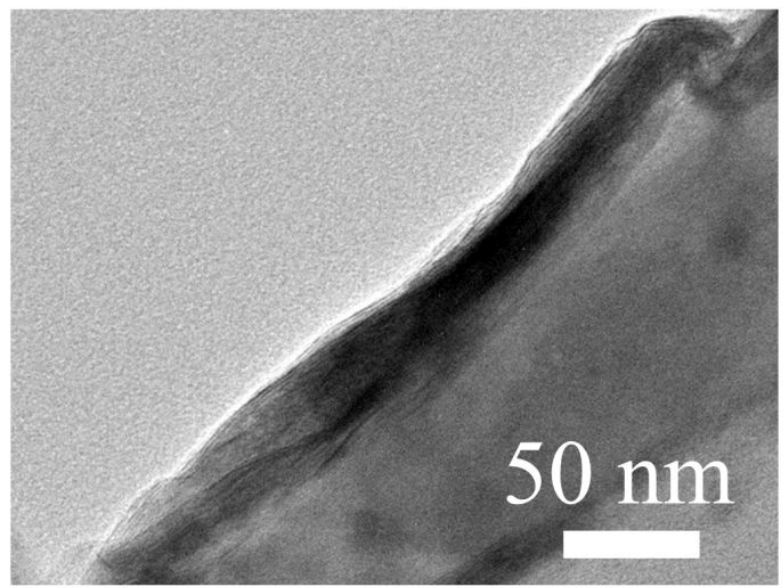

Figure S4. Cross-section TEM images of the M-fabric.

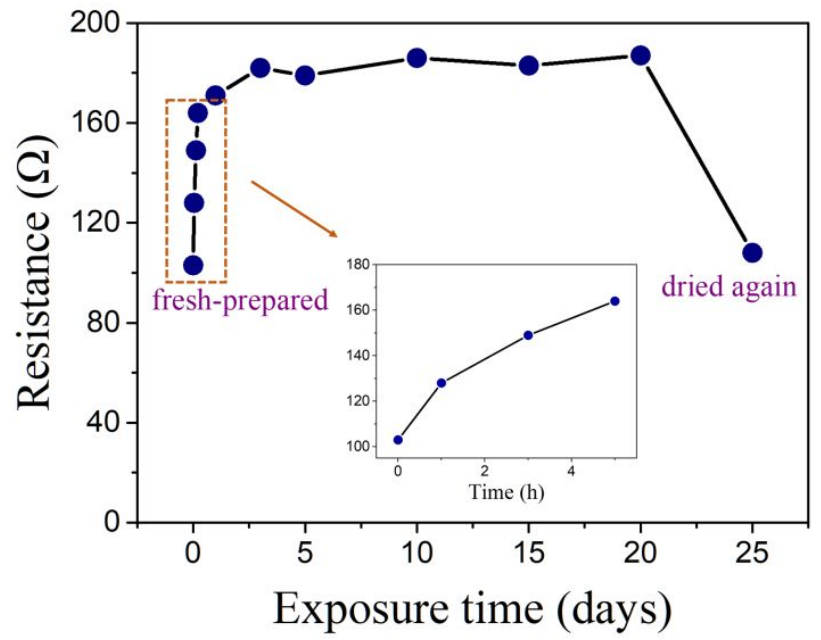

Figure S5. The resistance evolution of M-fabric after different exposure time under ambient humidity condition.

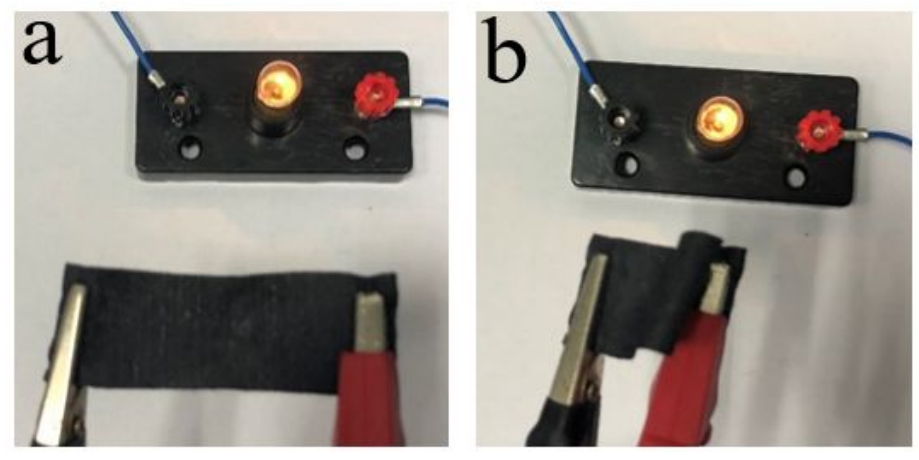

Figure S6. The digital photographs of an electric circuit with M-fabric when the M-fabric is in the straight (a) and bent (b) states. 


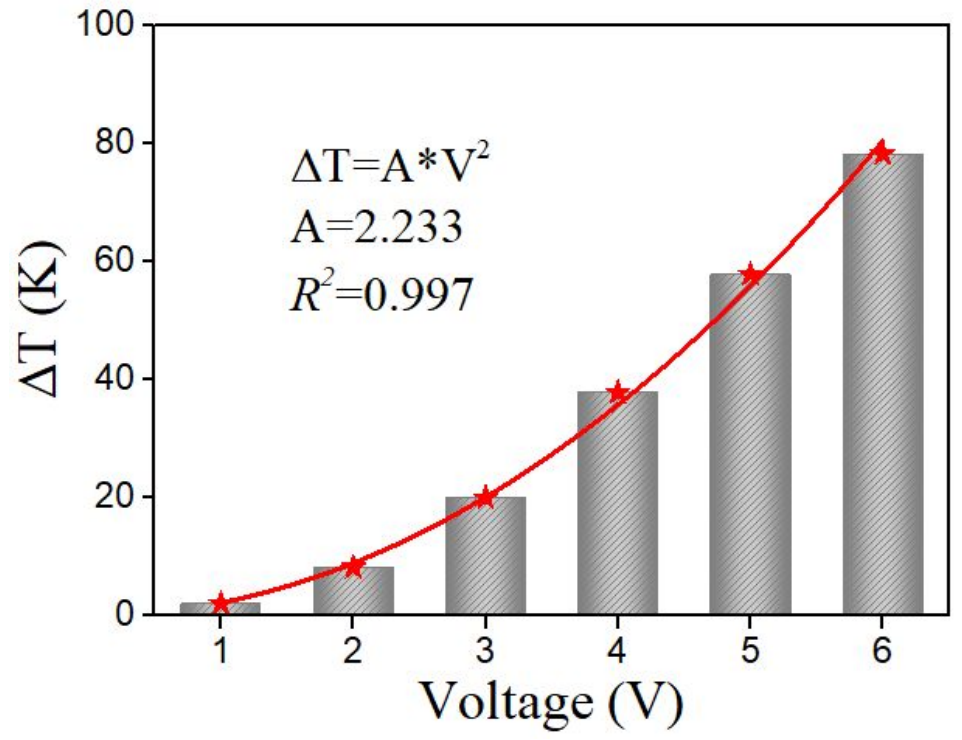

Figure S7. The temperature increase of the M-fabric under different applied voltages and corresponding fitting result.

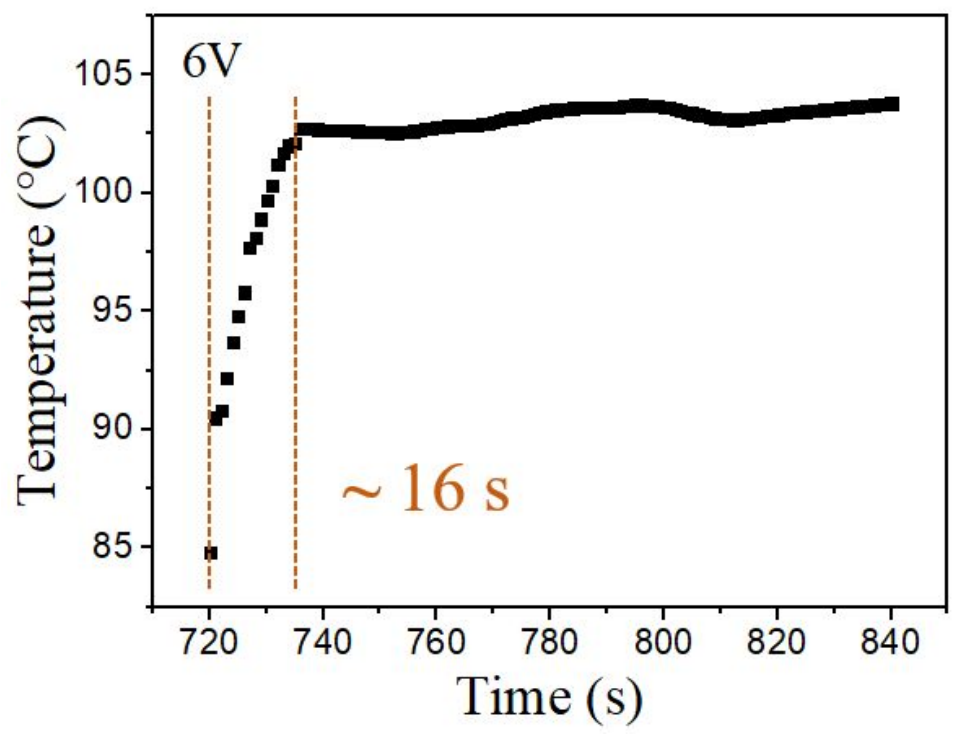

Figure S8. Temperature profile of M-fabric at applied voltage of $6 \mathrm{~V}$ showing the fast heating process. 

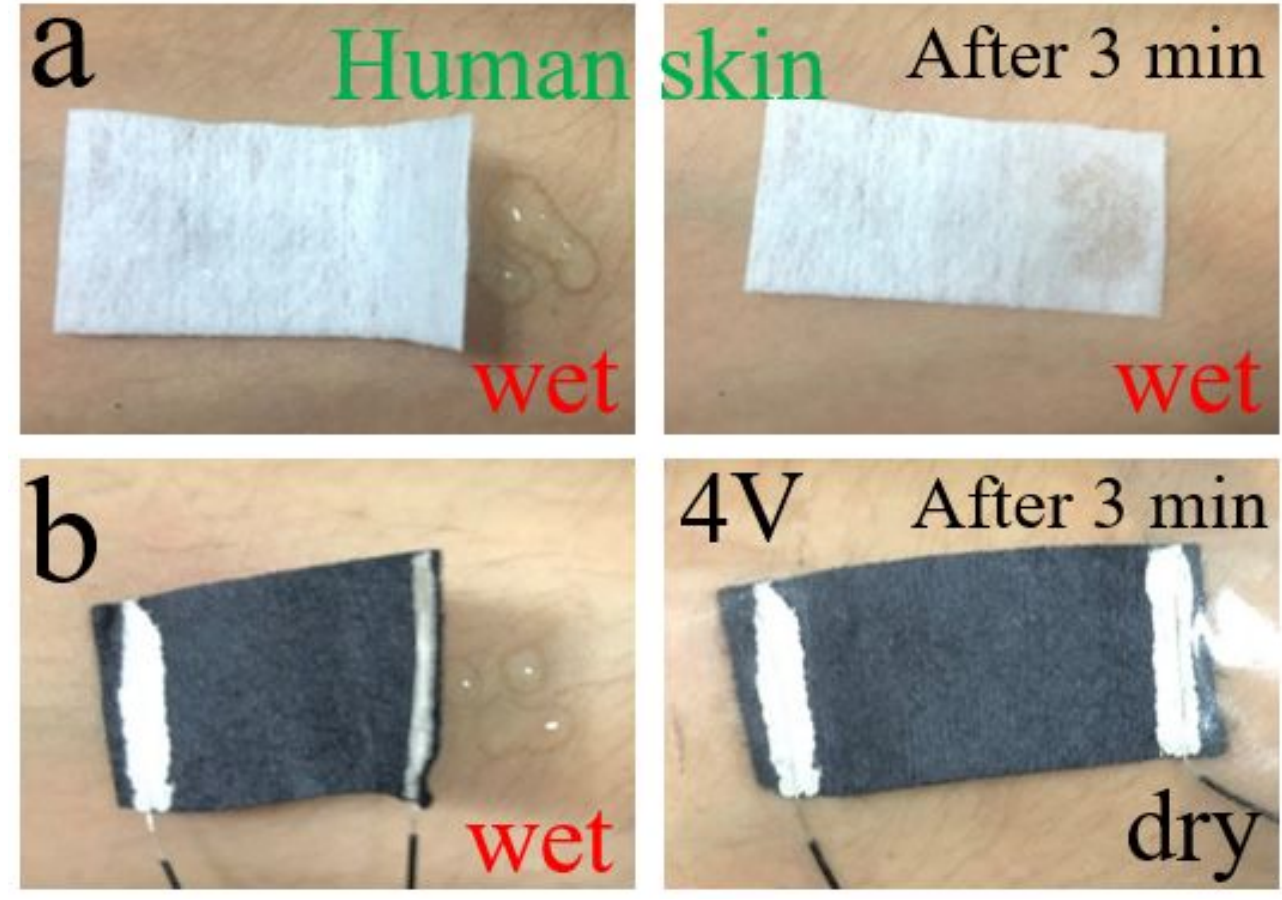

Figure S9. Digital photographs showing the slow-dry process of the pristine cellulose fabric (a) and the quick-dry performance of M-fabric (b) under an applied voltage of $4 \mathrm{~V}$.

a
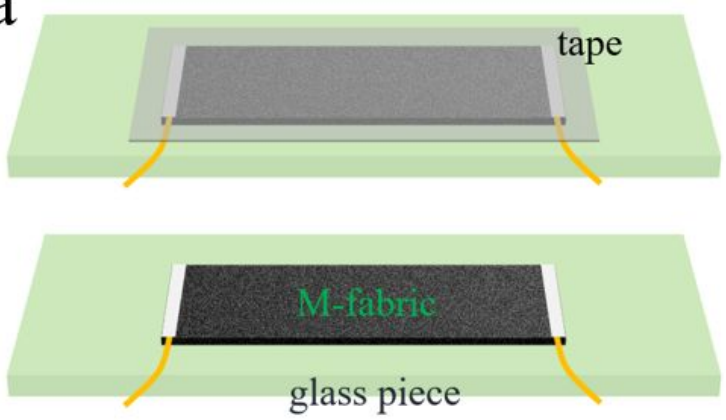

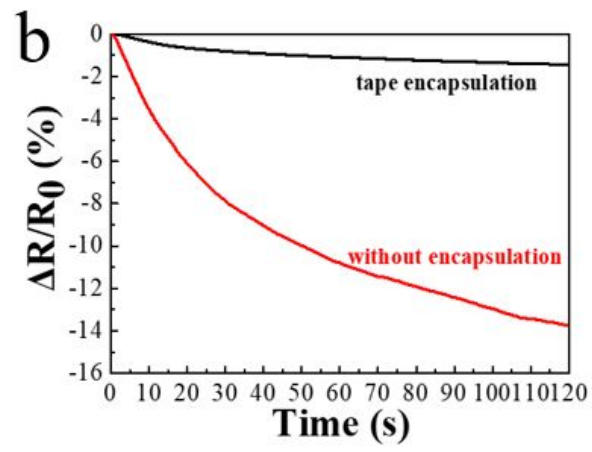

Figure S10. (a) Schematic illustration of M-fabric encapsulated by tape. (b) The relative resistance changes of M-fabric according to time upon voltage supply with and without tape encapsulation. 

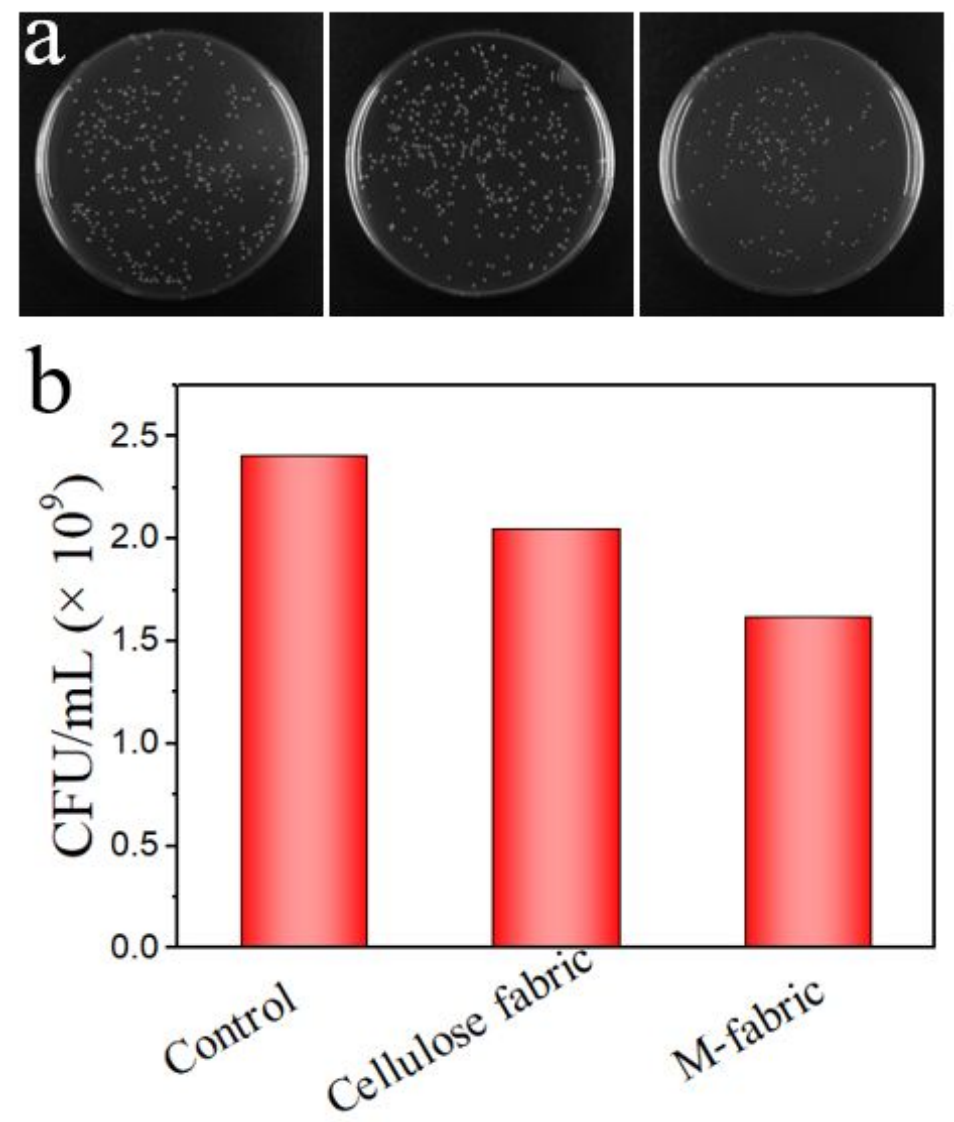

Figure S11. Representative images of colony forming units (a) and the numbers of live bacteria (b) for $S$. aureus suspensions by spreading their media on LB agar plates following $24 \mathrm{~h}$ incubation at $37^{\circ} \mathrm{C}$. 